CIENCIA Y SOCIEDAD

Volumen XXX, Número 2

Abil-Junio 2005

\title{
LA ESTABILIDAD DEL MERCADO DE ELECTRICIDAD DE REPÚBLICA DOMINICANA
}

\section{Juan Carlos Fabián Janampa*}

\begin{abstract}
RESUMEN
Se formulan relaciones con ecuaciones diferenciales que reflejan la dinámica del mercado. A partir de ellas se determinan los valores propios o eigenvalores para determinar en qué casos el mercado de electricidad se hace inestable y cuáles son las variables más incidentes en la inestabilidad.
\end{abstract}

\section{PALABRAS CLAVE}

Estabilidad económica, eigenvalores, mercado de contratos y mercado spot, pérdidas de distribución, regulación de la distribución.

\section{Introducción}

El diseño del sector electricidad de República Dominicana se fundamentó sobre la experiencia desregulatoria sudamericana, segmentados en actividades de generación, transporte y distribución. existen dos mercados: el de contratos y el mercado spot, en el mercado mayorista el distribuidor puede efectuar compras en el mercado spot con una restricción de un porcentaje determinado en función a sus contratos y su

* jcfabianj@hotmail.com 
máxima demanda. La característica de la reforma contaba con una participación del estado de $50 \%$ en las nacientes empresas de generación y distribución, y existían contratos entre el Estado con productores independientes. Los distribuidores comprarían energía en el mercado de contratos en la modalidad de licitación. Bajos índices de cobranza, elevados niveles de pérdidas en el segmento distribución y debilidades regulatorias caracterizan el sector, que lo hacen inestable financieramente.

\section{La dinámica de un simple sistema económico ${ }^{1}$}

Si se asume que un agente generador incrementa su producción $\left(P_{g}\right)$ siempre y cuando el precio del mercado $(\lambda)$ sea mayor que su costo marginal $\left(\lambda_{g}\right)$, el generador expandirá su producción hasta que iguale el costo marginal, la tasa de expansión es proporcional a la diferencia del precio de mercado y el costo marginal, podemos escribir entonces la siguiente ecuación diferencial:

$$
\tau_{g} \frac{d P_{g}}{d t}=\lambda-\lambda_{g}
$$

Donde :

$$
\lambda_{g}=b_{g}+c_{g} P_{g}
$$

Así también el consumidor incrementa su consumo $\left(\mathrm{P}_{\mathrm{d}}\right)$ siempre que su beneficio marginal $\left(\lambda_{d}\right)$ sea mayor que el precio de mercado $(\lambda)$, entonces la tasa de crecimiento de su consumo es proporcional a la diferencia de su beneficio marginal respecto al costo marginal. 
$\tau_{\mathrm{d}} \frac{d P_{\mathrm{d}}}{d t}=\lambda_{\mathrm{d}}-\lambda$

Donde:

$\lambda_{d}=b_{d}+c_{d} P_{d}$

Lo consumido es igual a lo generado.

$P_{g}=P_{d}$

Las ecuaciones se resumen en:

$\tau_{g} \frac{d P_{g}}{d t}=\lambda-b_{g}-c_{g} P_{g} \ldots \ldots \ldots$

$\tau_{d} \frac{d P_{d}}{d t}=b_{d}+c_{d} P_{d}-\lambda$

$P_{g}=P_{d}$

En forma matricial

$$
\left[\begin{array}{lll}
\tau_{g} & 0 & 0 \\
0 & \tau_{d} & 0 \\
0 & 0 & 0
\end{array}\right]\left[\begin{array}{l}
P_{g} \\
\dot{P}^{\prime} \\
P_{d} \\
\dot{\lambda}^{\prime}
\end{array}\right]=\left[\begin{array}{lll}
-c_{g} & 0 & -1 \\
0 & c_{d} & -1 \\
1 & -1 & 0
\end{array}\right]\left[\begin{array}{l}
P_{g} \\
P_{d} \\
\lambda
\end{array}\right]+\left[\begin{array}{l}
-b_{g} \\
b_{d} \\
0
\end{array}\right]
$$

Este sistema de ecuaciones puede simplificarse reemplazando la ecuación (6) en (5) y sumando las ecuaciones (4) y (5) modificada. 
La condición que debe cumplirse para garantizar estabili$\mathrm{dad}^{1}$ en este sistema es que:

$$
\frac{c_{g}-c_{d}}{\tau_{g}+\tau_{d}}>0
$$

Dada las constantes de tiempo $\tau_{g}$ y $\tau_{d}$ mayores que cero entonces:

$$
c_{g}>c_{d}
$$

Significa que la pendiente del costo marginal de generador debe ser mayor que la pendiente del beneficio marginal del consumidor.

El gráfico $\mathrm{N}^{\circ} 1$ es un ejemplo de sistema estable. El costo marginal de producción crece con la producción $\left(c_{g}>0\right)$, mientras el beneficio marginal del consumidor decrece con el consumo $\left(c_{d}<0\right)$.

\section{Gráfico $N^{\circ} 1$}

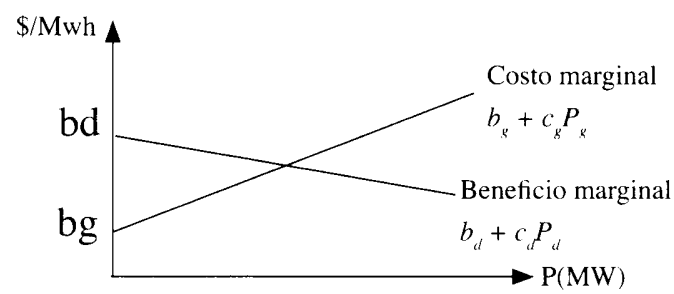

En ocasiones $c_{g}<0$ es decir el costo marginal decrece con el incremento de la producción, ello debido a economías de escala, o por regímenes de operación de algunas unidades en

1 Un sistema de ecuaciones diferenciales de la forma $\dot{X}=A X+B$ presenta solución del tipo $X=c_{1} \mathrm{e}^{\gamma_{1} t}+\mathrm{c}_{2} \mathrm{e}^{\gamma_{2} t}+$.donde los $\gamma$ son los eigenvalores o valores propios de la matriz $\mathrm{A}$. Los eigenvalores pueden ser números reales o complejos y resultan de hacer igual a cero el determinante de la matriz $[\mathrm{A}-\gamma \mathrm{I}]$ donde I es la matriz identidad. Para garantizar la estabilidad, debe cumplirse que la parte real de todos los $\gamma$ sea menor que cero. 
su mínimo técnico, o por que se ha fijado un costo marginal máximo que es inferior a la unidad de mayor costo variable disponible en el sistema.

Estos hechos son muy recurrentes en República Dominicana y constituyen errores regulatorios.

Con la exposición de este simple sistema económico extendemos una formulación de la dinámica de la realidad del sistema eléctrico de República Dominicana

\section{La dinámica del Mercado de Electricidad de República Dominicana}

Consideramos para este caso períodos mensuales de operación y nos enfocamos en el costo de la energía y la potencia, no incluimos los cargos por transmisión por simplicidad. Además si se incluyen, no modificarán sustancialmente los resultados que se obtienen. Asumimos también que el segmento de clientes libres que participan en el mercado mayorista es pequeño comparado con la demanda de los distribuidores.

En el mercado un generador inyectará energía mientras sus ingresos sean mayores que sus costos de operación. La tasa de crecimiento de la energía inyectada es proporcional al beneficio que obtiene.

$$
\tau_{g} \frac{d E_{g}}{d t}=\text { Ingresos }_{g}-\text { Costos }_{g}
$$

así también un distribuidor consumirá energía en proporción a la diferencia de sus ingresos con sus costos de operación.

$$
\tau_{g} \frac{d E_{d}}{d t}=\text { Ingresos }_{d}-\text { Costos }_{d}
$$




\section{Ingresos del generador:}

El ingreso está dado por las ventas de energía a sus contratos valorizados a sus precios de contrato, más las ventas de sus excedentes en el mercado spot valorizados a costo marginal, más sus ingresos por potencia en el mercado spot y en el de contratos.

$$
\left.P C_{g d} \times E_{\text {contratadag }}+C M G \times\left(E_{\text {generada }}-E_{\text {contratadag }}\right)+I_{\text {potenciag }}\right)
$$

\section{Costos del generador}

Los costos que incurre un generador están dados por el consumo de combustible más los no combustibles y otros costos de operación.

$$
C V_{g} \times E_{\text {generada }}+C O_{g}
$$

\section{Ingresos del distribuidor}

El ingreso del distribuidor es su cobranza que es igual a la energía comprada menos las pérdidas valorizadas con la tarifa de energía de la distribución.

$$
\text { Tarifa x }\left(E_{\text {consumida }}-E_{\text {pdistrib }}\right)
$$

\section{Costos de distribución}

Los costos que se incurren en distribución son los costos de compra de energía y potencia a los generadores en el mercado de contratos y en el mercado spot más los costos de operación y mantenimiento. Ello es igual a la energía contratada valorizada al precio de contrato de energía adicionado con la compra de su déficit en el mercado spot valorizado a costo marginal más los pagos por potencia y los costos de operación y mantenimiento.

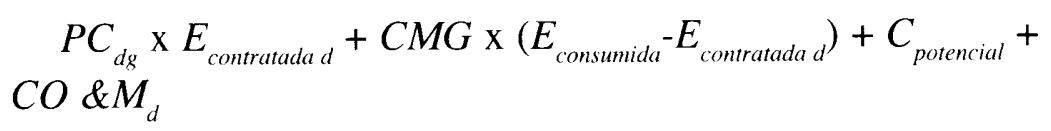




\section{Pérdidas de Distribución}

Entendemos como pérdidas de distribución todo lo que el distribuidor no puede efectivamente cobrar como son las pérdidas técnicas y no técnicas entre estas últimas el hurto, la morosidad, etc.

Asumiremos que las pérdidas tienen una naturaleza exponencial y es función también de la tarifa y de la energía comprada por el distribuidor. Se ha comprobado que si la tarifa sube existen mayores pérdidas no técnicas, así también si la generación es menor la morosidad se incrementa.

Es razonable proponer que la tasa de crecimiento de las pérdidas tiene la siguiente estructura:

$$
\tau_{p} \frac{d E_{p d i s t r i b}}{d t}=k_{p} E_{p d i s t r i b}+k_{p}^{\prime} \text { Tarifa } E_{\text {consumida }}+k
$$

$k_{p}$ representa el esfuerzo que hace el distribuidor para reducir sus pérdidas y representa el costo de reducir dichas pérdidas (US\$/MWh), $k_{p}^{\prime}$ representa el comportamiento natural de la población ante la calidad y el precio del suministro y a su vez representa la variación de las pérdidas técnicas en las redes de distribución (cuando se incrementa la demanda las pérdidas técnicas en la distribución tienden a incrementarse), $\mathrm{k}$ es una constante que asocia el valor inicial de las pérdidas de distribución.

\section{Balance de energía y pérdidas en la red de transmisión}

Se debe cumplir también que la energía inyectada por los generadores es igual a la energía consumida por el distribuidor más las pérdidas en la red de transporte de alta tensión.

$$
E_{g}=E_{d}+E_{p t r a n s}
$$


Las ecuaciones que gobiernan la dinámica del mercado representando todo el bloque generador en un solo agente y todo el bloque distribuidor también en otro agente son:

$$
\begin{aligned}
& \tau_{p} \frac{d E_{g}}{d t}=P C_{g d}+E_{\text {contratadag }}+C M G \times\left(E_{g}-E_{\text {contratadag }}\right) \\
& +I_{\text {potencing }}-C V_{g} \times E_{g}-C O_{g} \\
& \tau_{d} \frac{d E_{d}}{d t}=\text { Tarifa }_{d} \times\left(E_{d}-E_{p d i s t r i b}\right)-P C_{d g} \times E_{\text {contratadag }}-C M G \times \\
& \left(E_{d}-E_{\text {contratadad }}\right)-C_{\text {potenciad }}-\operatorname{CO\& } M_{d} \\
& \tau_{p} \frac{d E_{p d i s t r i b}}{d t}=k_{p} E_{p d i s t r i b}+k_{p}^{\prime} \operatorname{Tarifa}_{d}+k_{c} \\
& E_{g}=E_{d}+E_{\text {ptrans }}
\end{aligned}
$$

Como analizamos en términos globales podemos asumir un único precio de contrato de compra y venta de energía PC (es decir $P C_{g d}=P C_{d g}$ ) y una única Energía contratada contratada $\mathrm{E}_{\text {contratadad }}\left(\mathrm{E}_{\text {contratadag }}=\mathrm{E}_{\text {contratadad }}\right.$ ), así también que los ingresos por potencia del generador son independientes de las variables y son iguales a los pagos por potencia de la distribución $\mathrm{P}\left(I_{\text {potenciag }}=C_{\text {potenciad }}\right)$, entonces en términos matriciales podemos organizar esas ecuaciones como:

$$
\left[\begin{array}{llll}
\tau_{g} & 0 & 0 & 0 \\
0 & \tau_{d} & 0 & 0 \\
0 & 0 & \tau_{p} & 0 \\
0 & 0 & 0 & 0
\end{array}\right]\left[\begin{array}{c}
\dot{E}_{g} \\
\dot{E}_{d} \\
\dot{\bullet}_{p d i s t r i b} \\
E_{\text {contratada }}
\end{array}\right]=
$$


$=\left[\begin{array}{cccc}C M G-C V_{g} & 0 & 0 & P C-C M G \\ 0 & \text { Tarifa-CMG } & - \text { Tarifa } & C M G-P C \\ 0 & k_{p}^{\prime} \text { Tarifa } & k_{p} & 0 \\ 1 & -1 & 0 & 0\end{array}\right]\left[\begin{array}{c}E_{g} \\ E_{d} \\ E_{p d i s t r i b} \\ E_{\text {contratada }}\end{array}\right]+$

$+\left[\begin{array}{c}P-C O_{g} \\ -P-C O \& M_{d} \\ k \\ -E_{p t r a n s}\end{array}\right]$

Es oportuno mencionar que el $\mathrm{CV}$ es una función de la $\mathrm{E}_{\mathrm{g}}$. Por simplicidad asumiremos que existe un $\mathrm{CV}$ ponderado que representa los costos combustibles y no combustibles.

Reemplazando la ecuación (10) en la (8) y (9) y sumando luego la (7) y (8) modificada, tenemos el siguiente sistema de ecuaciones

$$
\begin{aligned}
& {\left[\begin{array}{cc}
\tau_{g}+\tau_{d} & 0 \\
0 & \tau_{p}
\end{array}\right]\left[\begin{array}{c}
\bullet \\
E_{g} \\
E_{p d i s t r i b}
\end{array}\right]=\left[\begin{array}{ll}
\text { Tarifa-CV } & - \text { Tarifa } \\
k_{p}^{\prime} \text { Tarifa } & k_{p}
\end{array}\right]\left[\begin{array}{c}
E_{g} \\
E_{p d i s t r i b}
\end{array}\right]+} \\
& +\left[\begin{array}{c}
(C M G \text {-Tarifa }) E_{p t r a n s}-C O-C O \& M_{g} \\
k-k_{p}^{\prime} \text { Tarifa } E_{\text {prans }}
\end{array}\right]
\end{aligned}
$$


Nótese en este caso que el precio y la energía contratada no intervienen directamente en la evaluación, éstas se simplifican en las ecuaciones.

Ahora observaremos el punto inicial de las variables de estado de este sistema. Para ello hacemos igual a cero el componente izquierdo que contiene las derivadas, de la última ecuación matricial. Tenemos entonces

$$
\begin{aligned}
& {\left[\begin{array}{cc}
\text { Tarifa-CV } & - \text { Tarifa } \\
k_{p}^{\prime} \text { Tarifa } & k_{p}
\end{array}\right]\left[\begin{array}{c}
E_{g} \\
E_{p d i s t r i b}
\end{array}\right]=} \\
& {\left[\begin{array}{c}
\left(\text { Tarifa-CMG) } E_{p t r a n s}+C O_{g}+C O \& M_{g}\right. \\
k_{p}^{\prime} \text { Tarifa } E_{p t r a n s}-k
\end{array}\right]}
\end{aligned}
$$

De ello resulta que la energía generada en el sistema y las pérdidas de distribución iniciales son iguales a:

$$
\begin{aligned}
& E_{g}=\frac{\left(\text { Tarifa-CMG }+\frac{k_{p}^{\prime}}{k_{p}} \text { Tarifa }^{2}\right) E_{\text {prans }}+C O_{g}+C O \& M-\frac{k}{k_{p}} \text { Tarifa }}{\text { Tarifa } C V+\frac{k_{p}^{\prime}}{k_{p}} \text { Tarifa }^{2}} \\
& E_{p d i s s r i b}=\frac{\frac{k_{p}^{\prime}}{k_{p}} \operatorname{Tarifa}(C M G-C V) E_{p t r a n s}-\frac{k_{k}}{k_{p}}(\operatorname{Tarifa}-C V)-\frac{k_{p}^{\prime}}{k_{p}} \operatorname{Tarifa}\left(C O_{g}+C O \& M^{d}\right)}{\operatorname{Tarifa-CV}+\frac{k_{p}^{\prime}}{k_{p}} \operatorname{Tarifa}^{2}}
\end{aligned}
$$


Analicemos ahora la estabilidad del sistema. Los eigenvalores o valores propios de este sistema de ecuaciones son:

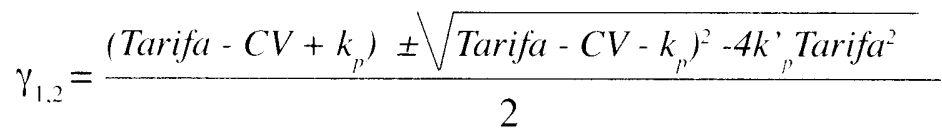

Debemos precisar que $k^{\prime}$, es una constante que es adimensional, mientras que $k_{p}$ tiene unidades de $(\$ / \mathrm{MWh})$.

Existen dos variantes:

\section{a) Eigenvalores sin parte imaginaria}

Debe cumplirse que:

$\left(\text { Tarifa-CV- } k_{p}\right)^{2} \geq 4 k_{p}^{\prime}$ Tarifa ${ }^{2}$

Así también para que exista estabilidad:

Tarifa-CV $+k_{p}<0 \mathrm{y}$

$a b s \mid\left(\right.$ Tarifa-CV $\left.+k_{p}\right) \mid>\sqrt{\left(\text { Tarifa-CV- } k_{p}\right)^{2}-4 k_{p}^{\prime} \text { Tarifa }^{2}}$

\section{b) Eigenvalores con parte imaginaria}

En este caso se cumple que:

$\left(\text { Tarifa-C }-k_{p}\right)^{2}<4 k_{p}^{\prime}$ Tarifa $^{2}$

y para que sea estable se tiene que garantizar también que:

Tarifa $-C V+k_{p}<0$ 
De todo lo anterior las inecuaciones que rigen la estabilidad del sistema son:

$$
\begin{aligned}
& k_{p}<0 \\
& k_{p}^{\prime}>0 \\
& \text { Tarifa }<C V-k_{p} \\
& \text { Tarifa }-C V+\frac{k_{p}^{\prime}}{k p} \text { Tarifa }^{2}<0
\end{aligned}
$$

Entonces $k_{p}$ debe ser menor que cero, las pérdidas deben ser decrecientes, $k_{p}$, mayor que cero, significa que siempre las pérdidas de distribución se incrementan con el consumo y por ende con la generación del sistema. La tarifa debe ser menor que la suma del costo variable ponderado de producción con el costo unitario que representa reducir las pérdidas de distribución. Al inicio de todo proceso de "Reducción de Pérdidas" el costo para reducir cada unidad de energía de pérdidas es menor y se va incrementado hasta que se alcanza el nivel cercano a las pérdidas óptimas en la que se requiere de mayores inversiones para continuar reduciendo las pérdidas lo cual ya no resulta rentable. En este punto el costo unitario de reducir una unidad de energía de pérdidas es sumamente elevado.

$k_{p}^{\prime}$ representa el comportamiento de la población ante el incremento de la tarifa y la energía generada en el sistema, así también refleja el incremento de las pérdidas técnicas. Si la energía generada se incrementa, la población es incentivada a efectuar sus pagos puntualmente. El objetivo es mantener $k_{p}^{\prime} \approx 1$ que significa que a pesar de las variaciones de la tarifa la población paga sus facturas y solamente este factor reflejaría el incremento de las pérdidas técnicas. 


$$
\text { Nótese }{ }^{2} \text { también que Tarifa }-C V=\frac{k_{p}^{\prime}}{k_{p}} \operatorname{Tarifa}^{2}<0
$$

Conclusiones fundamentales se extraen de estos simples resultados:

La tarifa debe reflejar los costos de operación exclusivamente y debe tener como límite máximo el valor de la suma del costo variable ponderado de producción y el costo de reducir las pérdidas de distribución.

Nótese que si se incluyen los precios de contrato para determinar la tarifa y éstos pueden ser mayores que los costos marginales, el sistema puede perder estabilidad.

Todo depende del nivel de pérdidas y las acciones que se realicen para reducirlas.

En muchos países la tarifa se fija reflejando los costos marginales esperados de la operación del sistema. El precio de contrato encontrado en el mercado actúa como un límite.

El otro factor fundamental que condiciona a la primera conclusión es que si las pérdidas del sistema no se reducen este sistema no será estable, si $k_{p}<0$, significa que las pérdidas tienen un comportamiento exponencial decreciente en el tiempo.

\section{Análisis numérico}

En el cuadro $\mathrm{N}^{\circ} 1$ se muestra el análisis de estabilidad para distintos escenarios. Se ha intentado reproducir algunas situaciones recurrentes en República Dominicana.

2 Los puntos iniciales de la energía generada y de las pérdidas dependen también del valor de $\mathrm{k}$, por lo general los puntos iniciales son positivos, se entiende que para ello $\mathrm{k}$ debe tener signo negativo. 
Se puede observar que:

Cuando no se reducen las pérdidas ( $k_{p}>0$ casos 1 y 3 ) el sistema es inestable. Cuando no se tiene un programa adecuado de reducción de pérdidas ( $k_{p}<0$ y de menor valor absoluto) y cuando el comportamiento de la población ante el alza de tarifas es influyente $\left(k_{p}^{\prime}<0\right.$ casos 2,4 y 5 ) el sistema es inestable.

Si la tarifa es mucho mayor comparada con la suma del costo de reducir pérdidas y el costo variable ponderado de generación ( Tarifa $>C V-k_{p}$, caso 6 ) por más que se reduzcan pérdidas no se alcanzará estabilidad.

El caso 7 representa un caso ideal en el que las pérdidas no dependen de la energía generada, el cual también resulta inestable.

En los casos 8,9 y 12 la tarifa es igual al costo variable más el costo de reducir pérdidas, el cual es inestable. El caso 18 en el que $k_{p}=0$ también es inestable.

Los otros casos son estables porque existe una reducción de pérdidas y se reflejan solamente variaciones de pérdidas técnicas.

$$
\left(k_{p}<0, k_{p}^{\prime}>0 \text { y Tarifa }-C V+\frac{k_{p}^{\prime}}{k_{p}} \text { Tarif }^{2}<0\right)
$$

Se observa también que un mercado en el que las pérdidas se reducen notablemente o tiene menores pérdidas $\left(-k_{p}\right.$ $>>0$ y $k_{0}^{\prime} \approx 1$ ) existe un gran margen entre el costo variable ponderado y la tarifa. Ello implica que existe mayor margen para negociar los precios de contrato de largo plazo entre generadordistribuidor así como un mayor valor agregado de distribución. 


\section{Cuadro $N^{\circ} 1$}

\begin{tabular}{|c|c|c|c|c|c|c|c|}
\hline Caso & $\begin{array}{c}\text { CV } \\
\text { Costo } \\
\text { variable } \\
\text { promedio } \\
\text { de pro- } \\
\text { ducción } \\
\text { (US\$/ } \\
\mathrm{MWh)}\end{array}$ & $\begin{array}{c}\text { kp } \\
\text { Costo de } \\
\text { reducir } \\
\text { pérdidas } \\
\text { (US\$/ } \\
\text { MWh) }\end{array}$ & $\begin{array}{c}\text { kp } \\
\text { Refleja } \\
\text { compor- } \\
\text { tamiento } \\
\text { de pérdi- } \\
\text { das con } \\
\text { tarifa y } \\
\text { energía } \\
\text { generada }\end{array}$ & $\begin{array}{l}\text { Tarifa } \\
\text { (US\$ } \$ \\
\text { MWh) }\end{array}$ & Eigenvalor 1 & Eigenvalor 2 & Estab. \\
\hline 1 & 40 & 20 & 0.01 & 120 & $77.5+j 0$ & $22.5+j 0$ & Inestable \\
\hline 2 & 70 & -20 & -0.50 & 60 & $27.7+j 0$ & $-57.7+j 0$ & Inestable \\
\hline 3 & 80 & 10 & 0.02 & 70 & $1.4+j 0$ & $-1.4+j 0$ & Inestable \\
\hline 4 & 40 & -20 & -0.01 & 120 & $81.4+j 0$ & $-214+j 0$ & Inestable \\
\hline 5 & 40 & -100 & .1 .00 & 120 & $140+j 0$ & $-160+i 0$ & lonestable \\
\hline 6 & 40) & -20 & 0.01 & 120 & $78.5+j 0$ & $-18.5+i 0$ & Incstable \\
\hline 7 & 40 & -20 & 0.00 & 120 & $8(1+j 0)$ & $-20+j 0$ & Inestable \\
\hline 8 & 40 & -80 & 0.01 & 120 & $79.1+j 0$ & $.79 .1+j 0$ & Inestable \\
\hline 9 & 40 & -80 & 0.50 & 1200 & $0+j 110.0$ & $0-j 116.6$ & Inestable: \\
\hline 10 & 40 & -81 & 1.00 & 120 & $-0.5+j 1+4.5$ & $0.5-j 144.5$ & Estable \\
\hline 11 & 40 & -100 & 1.00 & 120 & $.10+j 150$ & $-10-j 150$ & Estable \\
\hline 12 & 50 & -70 & 1.00 & 120 & $0+j 138.9$ & $0-j 1380$ & Inestable: \\
\hline 13 & 50 & -90 & 0.50 & 120 & $-10+j 116.6$ & $-10-j 116.0$ & Fstable \\
\hline 14 & 40 & -70 & 0.43 & 100 & $-5+j 92.3$ & $-5-j 92.3$ & Fistable \\
\hline 15 & 40 & -80 & 0.50 & 100 & $-10+j 99.5$ & $-10-j 99.5$ & Estable \\
\hline 16 & 40 & -140 & 0.75 & 140 & $-20+j 170.6$ & $-20-j 170.6$ & Estable \\
\hline 17 & 40 & -160 & 0.90 & 180 & $-10+j 227.3$ & $-10-j 227.3$ & Estable \\
\hline 18 & 40 & 0 & 0.01 & 80 & $38.3+j 0$ & $1.7+j 0$ & Inestable \\
\hline
\end{tabular}

\section{Conclusiones}

- Se ha representado la dinámica económica del sector electricidad de República Dominicana desde el punto de vista de la energía a través de ecuaciones diferenciales.

- Se ha determinado un margen de estabilidad del mercado de electricidad de República Dominicana teniendo como variable la tarifa de electricidad. 
- Para alcanzar estabilidad se deben cumplir dos condiciones:

i) La tarifa debe ser menor que la suma del costo variable ponderado de generación y el costo de reducir pérdidas de distribución. Se incluyen en estas últimas pérdidas técnicas y no técnicas.

ii) Las pérdidas deben tener un comportamiento decreciente en el tiempo.

- Un modelo más preciso nos indicaría que debieran reflejar los costos marginales en lugar del costo variable ponderado. No se ha incluido una función del beneficio que aportan los contratos, sin embargo dada la precariedad de la confiabilidad del sistema éste no sería apreciable. Nótese que a pesar de haber considerado el mercado de contratos en la formulación, el precio y la energía contratada se anulan y no participan directamente en el proceso de determinar la estabilidad del mercado.

- El límite de la tarifa está dado por la suma del costo variable ponderado de generación y el costo de reducción de pérdidas de distribución. Las pérdidas de distribución tienen que ser decrecientes en el tiempo. Si se plantea el diseño de un esquema tarifario que incluya en la tarifa los precios de contrato entre generador-distribuidor debiera considerarse este límite. Precios de contrato elevados pueden ocasionar que el mercado resulte inestable.

- En sistemas con mayor maduración o aquellos que no tienen pérdidas apreciables existe una gran brecha entre el costo variable y la tarifa, que permite un mayor margen de negociación de los precios de contrato, pero en aquellos en los que los niveles de pérdidas son elevados no se pueden establecer tarifas elevadas. 
- El diseño de esquemas tarifarios debiera considerar análisis de estabilidad del mercado de electricidad.

\section{Referencias}

1. Fernando L. Alvarado. "The Dynamics of Power System Market". Report Number PSERC-97-01. www.pserc.wisc.edu/ecow/get/publicatio/1997 public/pserc9701.pdf

2. Reglamento de Electricidad de República Dominicana. www. oc.org.do

3. Andrés Fernández Díaz. "Dinámica Caótica en Economía". McGraw Hill. 2oEdición - 2000.

4. F. L. Alvarado. "The Stability of Power System Markets". IEEE Transactions on Power Systems, Vol. 14, No. 2, May 1999, pp. $505-511$

5. F. L. Alvarado. "Is System Control Entirely by Price Feasible?". Proceedings of the 36th Hawaii International Conference on System Sciences (HICSS'03) 2002 IEEE. 